\title{
BAER MODULES OVER DOMAINS
}

\author{
PAUL C. EKLOF, LASZLO FUCHS, AND SAHARON SHELAH
}

\begin{abstract}
For a commutative domain $R$ with 1 , an $R$-module $B$ is called a Baer module if $\operatorname{Ext}_{R}^{1}(B, T)=0$ for all torsion $R$-modules $T$. The structure of Baer modules over arbitrary domains is investigated, and the problem is reduced to the case of countably generated Baer modules. This requires a general version of the singular compactness theorem. As an application we show that over $h$ local Prüfer domains, Baer modules are necessarily projective. In addition, we establish an independence result for a weaker version of Baer modules.
\end{abstract}

\section{INTRODUCTION}

In his seminal paper $[\mathrm{B}]$ on mixed abelian groups, $\mathrm{R}$. Baer proved that a countable abelian group $B$ had to be free if $\operatorname{Ext}_{\mathbb{Z}}^{1}(B, T)=0$ for all torsion abelian groups $T$. The problem of determining the uncountable groups $B$ with this property turned out to be extremely difficult. Only 30 years later was it settled by Griffith [Gf] who showed that $B$ had to be free, no matter what its cardinality was. Nunke $[\mathrm{N}]$ and Grimaldi $[\mathrm{Gm}]$ generalized the results to modules over Dedekind domains. Recently, Göbel [Gö] showed that the results extend to torsion theories over Dedekind domains.

The problem of characterizing Baer modules $B$ over arbitrary domains $R$ (i.e., $R$-modules $B$ with $\operatorname{Ext}_{R}^{1}(B, T)=0$ for all torsion $R$-modules $T$ ) was raised by Kaplansky [K]. He established two useful lemmas that served as a starting point for the paper of two of the present authors [EF] in which a new approach was used to show that Baer modules over arbitrary valuation domains had to be free. While particular properties of modules over valuation domains were utilized to settle the case of countable rank, transfinite induction was needed for higher ranks. For regular cardinals, a lemma was proved which-in a more general form-will serve as a crucial lemma in the present paper. For singular cardinals, a version of Shelah's compactness theorem was used.

The point of departure for the present paper is the observation that the tools developed in $[E F]$ can be refined to deal with Baer modules over arbitrary do-

Received by the editors December 13, 1988.

1980 Mathematics Subject Classification (1985 Revision). Primary 13C05, 13G05, 13L05.

Key words and phrases. Baer module, flat and projective modules, continuous ascending chains, Prüfer domains, constructibility, Proper Forcing Axiom, singular compactness.

This research was partially supported by NSF Grants DMS-8600376 and DMS-8620379. 
mains. A stronger version of the compactness theorem on singular cardinals, which can settle the case of singular cardinals below the cardinality of the domain as well, is required. Our main theorem reads as follows:

Theorem A. A module $B$ over a domain $R$ is a Baer module if and only if there exists a well-ordered continuous ascending chain of submodules

$$
0=B_{0}<B_{1}<\cdots<B_{\nu}<\cdots<B_{\tau}=B \quad(\nu<\tau)
$$

for some ordinal $\tau$ such that, for each $\nu<\tau, B_{\nu+1} / B_{\nu}$ is a countably generated Baer module.

This result reduces the problem of characterizing Baer modules to the countably generated case. If $R$ is such that all countably generated Baer $R$-modules are projective (as is the case if $R$ is a P.I.D., a Dedekind domain, or a valuation domain), then our theorem implies that all Baer modules over $R$ are projective. (We do not know if countably generated Baer modules are projective for every domain $R$.)

We apply Theorem A to Prüfer domains and prove

Theorem B. A module over an h-local Prüfer domain is a Baer module exactly if it is projective.

Finally, we deal with an independence result. First we prove

Theorem C. Assume $V=L$. Let $R$ be any domain of cardinality $\leq \aleph_{1}$ and $M$ any $R$-module of projective dimension $\leq 1$. If $\operatorname{Ext}_{R}^{1}\left(M, T_{0}\right)=0$, where $T_{0}=\bigoplus_{\omega} \bigoplus_{0 \neq r \in R}(R / R r)$, then $M$ is a Baer module.

However, if we assume the Proper Forcing Axiom (PFA) rather than the Axiom of Constructibility $(\mathrm{V}=\mathrm{L})$, then Theorem $\mathrm{C}$ fails for any countable domain $R$ which is not a field.

The authors wish to thank Alan Mekler for helpful discussions.

\section{Preliminaries}

We start off with the following lemmas. For the proofs of Lemmas 1 and 2, see Kaplansky [K] or Eklof and Fuchs [EF].

Lemma 1. Baer modules over any domain are flat. They are therefore torsionfree.

Lemma 2. Baer modules have projective dimension $\leq 1$.

Call a submodule $N$ of an $R$-module $M$ with p.d. $M \leq 1$ a tight submodule if p.d. $M / N \leq 1$; then necessarily p.d. $N \leq 1$ as well. The following two lemmas were proved in Eklof and Fuchs [EF].

Lemma 3. Tight submodules of Baer modules are Baer modules.

Lemma 4. An R-module $B$ of projective dimension $\leq 1$ is a Baer module exactly if $\operatorname{Ext}_{R}^{1}(B, T)=0$ for all direct sums $T$ of cyclically presented torsion modules. 
Here by a cyclically presented $R$-module is meant one of the form $R / R r$ with $r \in R$.

Lemma 5. If an $R$-module $B$ of projective dimension $\leq 1$ can be generated by $\kappa$ elements ( $\kappa$ an infinite cardinal), then for $T_{0}=\bigoplus_{\kappa} \bigoplus_{0 \neq r \in R}(R / R r)$, $\operatorname{Ext}_{R}^{1}\left(B, T_{0}\right)=0$ implies that $B$ is a Baer module.

Proof. Let $0 \rightarrow H \stackrel{\alpha}{\rightarrow} F \rightarrow B \rightarrow 0$ be a projective resolution of $B$ where $F$ is a free $R$-module with $\kappa$ generators. Without loss of generality, we can assume $H$ is free, so likewise $\leq \kappa$-generated. Consider the induced sequence

$$
\operatorname{Hom}_{R}(F, T) \stackrel{a^{*}}{\rightarrow} \operatorname{Hom}_{R}(H, T) \rightarrow \operatorname{Ext}_{R}^{1}(B, T) \rightarrow 0
$$

where $T$ is a direct sum of cyclically presented torsion modules. Every homomorphism $\eta: H \rightarrow T$ lands in a $\kappa$-generated summand of $T$. Such a summand is isomorphic to a summand of $T_{0}$; thus, it is clear from the hypothesis on $T_{0}$ that $\eta$ must be induced by a homomorphism $F \rightarrow T$. We infer that $\alpha^{*}$ is epic and $\operatorname{Ext}_{R}^{1}(B, T)=0$. The preceding lemma completes the proof.

It is easy to show directly that over any domain $R$, a countably generated module of projective dimension $\leq 1$ has to be countably presented. Instead, we prove a result which is more useful for our purposes.

Proposition 6. Over any domain $R$, a countably generated flat module has projective dimension $\leq 1$ and is countably presented.

Proof. Let $M$ be a countably generated flat $R$-module and $0 \rightarrow H \rightarrow F \rightarrow$ $M \rightarrow 0$ a presentation of $M$ with $F$ countably generated free. Thus, $H$ is a pure submodule of $F$. Osofsky [O] showed that in a pure submodule of a free $R$-module ( $R$ any ring), every set of cardinality $\aleph_{k}$ can be embedded in a pure, $\aleph_{k}$-generated submodule and this submodule has p.d. $\leq k$. Applying this in our case to a maximal independent set in $H$, we conclude that $H$ is countably generated and p.d. $H=0$.

We can now derive

Corollary 7. Over any domain, countably generated Baer modules are countably presented.

Corollary 8. Pure submodules of countably generated Baer modules are Baer modules.

Proof. Let $H$ be a pure submodule in a countably generated Baer module $B$. Then $B / H$ is countably generated and flat, so by Proposition 6 p.d. $B / H \leq 1$. The claim follows from Lemma 3.

Our crucial lemma is the following; it generalizes Lemma 9 in [EF].

Lemma 9. Let $M$ be a $\kappa$-generated module ( $\kappa$ a regular uncountable cardinal), and suppose

$$
0=M_{0}<M_{1}<\cdots<M_{\alpha}<\cdots \quad(\alpha<\kappa)
$$


is a well-ordered continuous ascending chain of submodules such that

(i) $M_{\alpha}$ is less than $\kappa$-generated for each $\alpha<\kappa$;

(ii) p.d. $M_{\alpha+1} / M_{\alpha} \leq 1$ for each $x<\kappa$;

(iii) $\bigcup_{\alpha<\kappa} M_{\alpha}=M$.

For each $\alpha<\kappa$, let $G_{\alpha}$ be an $R$-module, and set $H_{\beta}=\bigoplus_{\alpha<\beta} G_{\alpha}(\beta<\kappa), H=$ $\bigoplus_{\alpha<\kappa} G_{\alpha}$. If the set

$$
E=\left\{\alpha<\kappa \mid \exists \beta>\alpha \text { with } \operatorname{Ext}_{R}^{1}\left(M_{\beta} / M_{\alpha}, H_{\beta} / H_{\alpha}\right) \neq 0\right\}
$$

is stationary in $\kappa$, then

$$
\operatorname{Ext}_{R}^{1}(M, H) \neq 0
$$

Proof. Dropping to a club if necessary, we may assume $\beta=\alpha+1$; condition (ii) continues to hold in view of a lemma by Auslander. Thus, it suffices to prove our claim under the hypothesis that $E=\left\{\alpha<\kappa \mid \operatorname{Ext}^{1}\left(M_{\alpha+1} / M_{\alpha}, G_{\alpha}\right) \neq 0\right\}$ is stationary in $\kappa$.

For each $0<\alpha<\kappa$ select an exact sequence

$$
0 \rightarrow G_{\alpha x} \rightarrow C_{\alpha} \stackrel{\rho_{a}}{\rightarrow} M_{\alpha+1} / M_{c r} \rightarrow 0
$$

which does not split when $\alpha \in E$. Following the pattern of [EF, Lemma 9], we construct for each $\alpha<\kappa$, an exact sequence of $R$-modules,

$$
0 \rightarrow H_{c r} \rightarrow X_{r} \stackrel{\phi_{i}}{\rightarrow} M_{c r} \rightarrow 0
$$

satisfying the following properties:

(1) $0=X_{0}<X_{1}<\cdots<X_{\alpha}<\cdots(\alpha<\kappa)$ is a well-ordered continuous ascending chain;

(2) the diagram

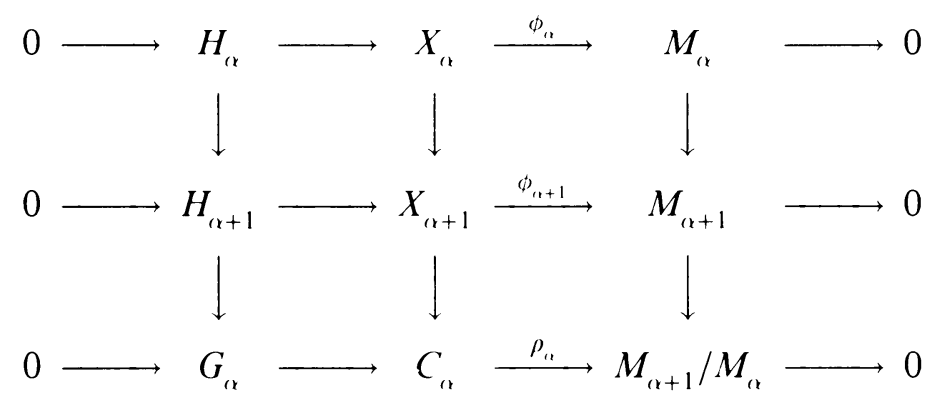

commutes for all $\alpha<\kappa$;

(3) for a limit ordinal $\beta<\kappa, 0 \rightarrow H_{\beta} \rightarrow X_{\beta} \stackrel{\phi_{\beta}}{\rightarrow} M_{\beta} \rightarrow 0$ is the direct limit of the exact sequences (2) with $\alpha<\beta$; 
(4) for all $\alpha<\beta<\kappa$, the diagrams

(4)

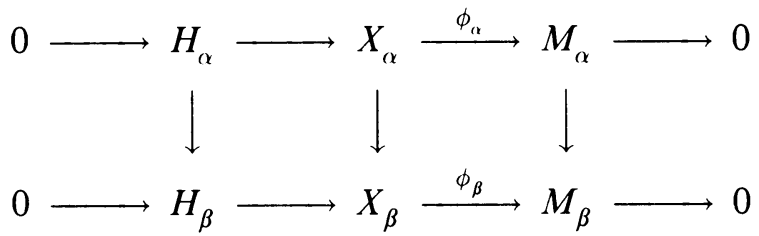

commute (the vertical maps are inclusions).

Define $0 \rightarrow H \rightarrow X \stackrel{\phi}{\rightarrow} M \rightarrow 0$ as the direct limit of the exact sequences (2) for $\alpha<\kappa$. The proof that this sequence does not split is identical to the one given at the end of the proof of Lemma 9 in [EF] (at the end of the proof, after concluding that the third row in the diagram splits, argue that the first vertical map is inclusion as a summand to obtain the splitting of the top row). Hence $\operatorname{Ext}_{R}^{1}(M, H) \neq 0$, indeed.

\section{SiNGULAR COMPACTNESS}

We will need a version of the Singular Compactness Theorem. This theorem says, roughly, that if $A$ is a structure of singular cardinality $\lambda$ such that "most" substructures of cardinality $<\lambda$ are "free", then $A$ is "free". The theorem applies to much more than the ordinary notion of freeness; for example, for us, "free" will mean the condition given as the conclusion of Theorem A. An axiomatic formulation of properties of "free" that are sufficient to prove the theorem is given in $[\mathrm{S}]$. Another axiomatic version is given in $[\mathrm{H}]$. In the version stated below, we follow closely the latter, except that we formulate it only for modules and state it so that it applies below the cardinality of the ring. (So we consider the cardinality of sets of generators of submodules rather than the cardinality of the submodules themselves.)

In the following $R$ is a fixed but arbitrary ring, and if $Y$ is a subset of an $R$-module $M,\langle Y\rangle$ denotes the submodule generated by $Y . \mathscr{P}(M)$ denotes the power set of $M$.

Suppose $\mathscr{F}$ is a class of $R$-modules such that for every $M \in \mathscr{F}$ we are given a nonempty set $\mathscr{B}(M) \in \mathscr{P}(\mathscr{P}(\mathscr{P}(M)))$. We assume that $\{0\} \in \mathscr{F}$ and that $\varnothing \in \mathscr{X}$ for all $\mathscr{X} \in \mathscr{B}(M)$. We also assume the following conditions hold for all $M \in \mathscr{F}$ and all $\mathscr{X} \in \mathscr{B}(M)$ :

(a) If $Y \in \mathscr{X}$, then $\langle Y\rangle \in \mathscr{F}$ and $\{Z \in \mathscr{X} \mid Z \subseteq\langle Y\rangle\} \in \mathscr{B}(\langle Y\rangle)$; in this case we say that $\langle Y\rangle$ is a free factor of $M$. Whenever $A$ is a free factor of $M$, we also suppose we are given a subset of $\mathscr{B}(A) \times \mathscr{B}(M)$; we write $\mathscr{X}^{\prime}=\mathscr{X} \mid A$ if $\left(\mathscr{X}^{\prime}, \mathscr{X}\right)$ belongs to this subset.

(b) If $A$ is a free factor of $M$ and $\mathscr{X}^{\prime}=\mathscr{X} \mid A$, then $\mathscr{X}^{\prime}=\{Z \in \mathscr{X}: Z \subseteq$ $A\}$; moreover, for every $\mathscr{X}^{\prime} \in \mathscr{B}(A)$, there exists $\mathscr{X} \in \mathscr{B}(M)$ such that $\mathscr{X}^{\prime}=\mathscr{X} \mid A$. 
(c) $\mathscr{X}$ is closed under unions of chains, i.e., if $C \subseteq \mathscr{X}$ such that for all $Y, Y^{\prime} \in C$, either $Y \subseteq Y^{\prime}$ or $Y^{\prime} \subseteq Y$, then $\cup C \in \mathscr{X}$.

(d) If $Y \in \mathscr{X}$ and $a \in M$, then there exists $Y^{\prime} \in \mathscr{X}$ such that $Y \subseteq Y^{\prime}$, $a \in\left\langle Y^{\prime}\right\rangle$, and $\left|Y^{\prime}\right| \leq|Y|+\aleph_{0}$; and

(e) If $M_{\nu} \in \mathscr{F}, \mathscr{X}_{\nu} \in \mathscr{B}\left(M_{\nu}\right)(\nu<\tau)$ are such that

$$
M_{0}<M_{1}<\cdots<M_{\nu}<\cdots
$$

is a well-ordered continuous ascending chain of submodules, $M_{\nu}$ is a free factor of $M_{\nu+1}$ and $\mathscr{X}_{\nu}=\mathscr{X}_{\mu} \mid M_{\nu}$ for all $\nu<\mu<\tau$, then $\bigcup_{\nu<\tau} M_{\nu}$ belongs to $\mathscr{F}$ and the closure $\mathscr{X}$ of $\bigcup_{\nu<\tau} \mathscr{X}$ under unions of chains belongs to $\mathscr{B}\left(\bigcup_{\nu<\tau} M_{\nu}\right)$.

The proof of the following version of the Singular Compactness Theorem can be found in the forthcoming book [EM2]; it follows closely the proof in [H].

Theorem 10. Let $\mathscr{F}$ satisfy (a)-(e). Suppose $\lambda$ is a singular cardinal, and $M$ is a $\lambda$-generated $R$-module. If for every $\kappa<\lambda$, there is a family $\mathscr{C}_{\kappa}$ of $\kappa$-generated submodules of $M$ such that:

$\left(1^{\prime}\right) \mathscr{C}_{\kappa} \subseteq \mathscr{F}$;

(2') $\mathscr{C}_{\kappa}$ is closed under unions of chains of length $\leq \kappa$; and

$\left(3^{\prime}\right)$ every subset $S$ of $M$ of cardinality $\leq \kappa$ is contained in a member of $\mathscr{C}_{\kappa}$,

then $M \in \mathscr{F}$.

For the simplest example of a family $\mathscr{F}$ satisfying the conditions (a)-(e), let $\mathscr{F}$ be the class of all free $R$-modules, and for each $M \in \mathscr{F}$, let $\mathscr{B}(M)$ be the set of all $\mathscr{X}$ such that there is a basis $B$ of $M$ with $\mathscr{Z}=\mathscr{P}(B)$. Then $A$ is a free factor of $M$ if and only if $A$ and $M / A$ are free; in that case let $\mathscr{X}^{\prime}=\mathscr{X} \mid A$ if and only if there is a basis $B$ of $M$ and a basis $B^{\prime}$ of $A$ such that $B^{\prime} \subseteq B, \mathscr{X}^{\prime}=\mathscr{P}\left(B^{\prime}\right)$, and $\mathscr{X}=\mathscr{P}(B)$.

Our application involves an $\mathscr{F}$ of the following type. Let $\mathcal{N}$ be a set of countably presented modules. (In our application $\mathscr{N}$ will be the set of all countably generated Baer modules; they are countably presented by Corollary 7.) Let $\mathscr{F}_{1}$. consist of all modules $M$ such that $M$ is the union of a continuous well-ordered ascending chain

$$
M_{0}<M_{1}<\cdots<M_{\nu}<\cdots<M_{\tau}=M
$$

where for each $\nu<\tau, M_{\nu+1} / M_{\nu} \in \mathcal{N}$. (We will refer to (5), in brief, as a "chain up to $M$ ".) For each $M \in \mathscr{F}_{A}$, let $\mathscr{B}(M)$ consist of all $\mathscr{X}$ such that there is a chain (5) satisfying: $Y \in \mathscr{X}$ if and only if

(6) for each $\nu<\tau,\left(\langle Y\rangle \cap M_{\nu+1}\right) \backslash M_{\nu} \neq \varnothing$ implies $M_{\nu+1} \subseteq M_{\nu}+\langle Y\rangle$.

Note that the chain (5) uniquely determines an $\mathscr{X} \in \mathscr{B}(M)$ according to (6), but there may be many representations of $M$ as the union of a chain (5), so $\mathscr{B}(M)$ may have many elements. If $A$ is a free factor of $M$, let $\mathscr{X}^{\prime}=\mathscr{X} \mid A$ if and only if: there is a chain (5) up to $M$ such that $A=M_{\mu}$ for some $\mu$; 
$\mathscr{X}$ is determined by the chain (5), as described in (6); and $\mathscr{X}^{\prime}$ is determined by the chain

$$
M_{0}<M_{1}<\cdots<M_{\mu}=A
$$

as in (6).

In order to be in a position to use Theorem 10 to prove Theorem A, we will verify

Theorem 11. With $\mathscr{F}_{\mathcal{N}}$ and $\mathscr{B}(M)$ as above, conditions (a)-(e) above are satisfied.

Proof. (a) If $Y \in \mathscr{B}(M)$ for some $M \in \mathscr{F}_{\mathscr{N}}$, then there is a sequence (5) such that (6) holds. Let $A=\langle Y\rangle$, and for each $\nu \leq \tau$, set $A_{\nu}=A \cap M_{\nu}$. Then clearly $A=\bigcup_{\nu<\tau} A_{\nu}$ and for each $\nu, A_{\nu+1} / A_{\nu}$ belongs to $\mathscr{N}$ because, by (6), it is either 0 or the canonical map

$$
A_{\nu+1} / A_{\nu} \rightarrow M_{\nu+1} / M_{\nu}
$$

is an isomorphism. It is clear that this chain $\left\{A_{\nu} \mid \nu \leq \tau\right\}$ determines the element $\{Z \in \mathscr{X} \mid Z \subseteq A\}$ of $\mathscr{B}(A)$. Thus (a) is satisfied.

(b) The first part of (b) is obvious. Now suppose $A$ is as in the proof of (a) and $\mathscr{X}^{\prime} \in \mathscr{B}(A)$. Thus there is a well-ordered continuous ascending chain

$$
B_{0}<\cdots<B_{\mu}<\cdots<B_{\rho}=A
$$

such that $B_{\mu+1} / B_{\mu} \in \mathscr{N}$ for all $\mu$ and $Y \in \mathscr{X}^{\prime}$ if and only if

$$
\text { for each } \mu<\rho,\left(\langle Y\rangle \cap B_{\mu+1}\right) \backslash B_{\mu} \neq \varnothing \text { implies } B_{\mu+1} \subseteq\langle Y\rangle+B_{\mu},
$$

We are now going to define by induction a well-ordered continuous ascending chain

$$
B_{0}<\cdots<B_{\mu}<\cdots<B_{\sigma}=B
$$

extending (7) such that: (i) for all $\mu<\sigma, B_{\mu+1} / B_{\mu} \in \mathcal{N}$; and (ii) for all $\mu \geq \rho$ and all $\nu<\tau,\left(B_{\mu} \cap M_{\nu+1}\right) \backslash M_{\nu} \neq \varnothing$ implies $M_{\nu+1} \subseteq B_{\mu}+M_{\nu}$. For $\mu \leq \rho$ we let $B_{\mu}$ be as already defined. Suppose that for some $\mu \geq \rho, B_{\mu}$ has been defined with the desired properties. Let $\gamma$ be minimal so that $M_{\gamma+1} \not \subset B_{\mu}$; thus by continuity, $M_{\gamma} \subseteq B_{\mu}$. (If there is none, then $B_{\mu}=M$ and we stop.) Set $B_{\mu+1}=B_{\mu}+M_{\gamma+1}$. Then the canonical map

$$
M_{\gamma+1} / M_{\gamma} \rightarrow B_{\mu+1} / B_{\mu}
$$

is well defined and onto. Moreover, it is one-one because by hypothesis (ii) on $B_{\mu}, M_{\gamma+1} \cap B_{\mu} \subseteq M_{\gamma}$ since $M_{\gamma+1} \not \subset B_{\mu}+M_{\gamma}=B_{\mu}$. Thus $B_{\mu+1} / B_{\mu} \cong$ $M_{\gamma+1} / M_{\gamma} \in \mathscr{N}$, and (i) is satisfied for $B_{\mu+1}$. It remains to verify (ii) for $B_{\mu+1}$. So suppose that for some $\nu,\left(B_{\mu+1} \cap M_{\nu+1}\right) \backslash M_{\nu} \neq \varnothing$. If $\nu \leq \gamma$, then $M_{\nu+1} \subseteq M_{\gamma+1} \subseteq B_{\mu+1}$, and we are done. So we may suppose that $\nu>\gamma$. We are assuming that there exist $b \in B_{\mu}, x \in M_{\gamma+1}$, and $u \in M_{\nu+1} \backslash M_{\nu}$ such that $b+x=u$. But then $b=-x+u \in M_{\nu+1} \backslash M_{\nu}$ and by hypothesis (ii) for $B_{\mu}$, $M_{\nu+1} \subseteq B_{\mu}+M_{\nu} \subseteq B_{\mu+1}+M_{\nu}$. 
For limit ordinals $\mu$ of course we define $B_{\mu}=\bigcup_{c<\mu} B_{\alpha}$. This completes the inductive definition of the $B_{\mu}$. If we define $\mathscr{X}$ to be the set of all $Y$ such that

$$
\text { for each } \mu<\sigma,\left(\langle Y\rangle \cap B_{\mu+1}\right) \backslash B_{\mu} \neq \varnothing \text { implies } B_{\mu+1} \subseteq\langle Y\rangle+B_{\mu},
$$

then $\mathscr{X}$ will be a member of $\mathscr{B}(M)$ and $\mathscr{X}^{\prime}=\mathscr{X} \mid A$. This completes the verification of $(b)$.

(c) It is obvious from the definition that $\mathscr{Z}$ is closed under unions of chains.

(d) In fact, we prove by induction on $\nu$ that given $Y \in \mathscr{X}$ and a countable $S \subseteq M_{\nu}$, there exists $Y^{\prime} \in \mathscr{X}$ such that $Y \subseteq Y^{\prime}, S \subseteq\left\langle Y^{\prime}\right\rangle$ and $\left|Y^{\prime}\right| \leq|Y|+\aleph_{0}$. Suppose that this has been proved for all $\nu<\gamma$.

Consider first the case when $\gamma$ is a limit ordinal; then each element of $S$ belongs to $M_{\nu}$ for some $\nu<\gamma$. Let $f: \omega \rightarrow S$ be an enumeration of $S$. Using the inductive hypothesis, define recursively $Y_{n} \in \mathscr{X}$ so that $Y_{0}=Y, Y_{n} \subseteq$ $Y_{n+1},\left|Y_{n+1}\right| \leq\left|Y_{n}\right|+\aleph_{0}$, and $f(n) \in\left\langle Y_{n+1}\right\rangle$. Then $Y^{\prime}=\bigcup_{n} Y_{n}$ contains $S$ and belongs to $\mathscr{X}$ since $\mathscr{X}$ is closed under unions of chains.

Now suppose $\gamma=\delta+1$. We consider two subcases. First, if $\langle Y\rangle \cap M_{\delta+1} \not \subset$ $M_{\delta}$, then by (6) $M_{\delta+1} \subseteq\langle Y\rangle+M_{\delta}$, so for each $a \in S$ there exists $y_{\alpha} \in\langle Y\rangle$ such that $a-y_{\alpha} \in M_{\delta}$. Let $S^{\prime}=\left\{a-y_{a} \mid a \in S\right\} \subseteq M_{\delta}$. By inductive hypothesis, there exists $Y^{\prime} \in \mathscr{X}$ containing $Y$ and such that $S^{\prime} \subseteq\left\langle Y^{\prime}\right\rangle$. Then also $S \subseteq\left\langle Y^{\prime}\right\rangle$ since $\langle Y\rangle \subseteq\left\langle Y^{\prime}\right\rangle$. Secondly, suppose $\langle Y\rangle \cap M_{\delta+1} \subseteq M_{\delta}$. It suffices to show that there exists $Y^{\prime} \supseteq Y$ such that $\left|Y^{\prime}\right| \leq|Y|+\aleph_{0}$ and $M_{\delta+1} \subseteq\left\langle Y^{\prime}\right\rangle+M_{\delta}$, for then we are reduced to the first subcase. Choose a countable subset $G$ of $M_{\delta+1}$ such that $M_{\delta+1}=\langle G\rangle+M_{\delta}$ and $\langle G\rangle \cap M_{\delta}=\left\langle G \cap M_{\delta}\right\rangle$; this is possible since $M_{\delta+1} / M_{\delta}$ is countably presented. By induction, there exists $Y^{\prime \prime} \in \mathscr{Z}$ such that $G \cap M_{\delta} \subseteq\left\langle Y^{\prime \prime}\right\rangle$ and $\left|Y^{\prime \prime}\right| \leq|Y|+\aleph_{0}$. If $\left\langle Y^{\prime \prime}\right\rangle \cap M_{\delta+1} \neq\left\langle Y^{\prime \prime}\right\rangle \cap M_{\delta}$, let $Y^{\prime}=Y^{\prime \prime}$; by (6) this implies that $M_{\delta+1} \subseteq\left\langle Y^{\prime}\right\rangle+M_{\delta}$. Otherwise let $Y^{\prime}=Y^{\prime \prime} \cup G$. It remains to show that this $Y^{\prime}$ belongs to $\mathscr{X}$. So suppose that for some $\mu$, there exist $y \in\left\langle Y^{\prime \prime}\right\rangle, g \in\langle G\rangle$ such that $y+g \in M_{\mu+1} \backslash M_{\mu}$; we must show that $M_{\mu+1} \subseteq\left\langle Y^{\prime}\right\rangle+M_{\mu}$. If $\mu=\delta$, this is the case by construction. If $\mu \geq \delta+1$, then $g \in M_{\delta+1} \subseteq M_{\mu}$, so $y \in M_{\mu+1} \backslash M_{\mu}$, and then since $Y^{\prime \prime} \in \mathscr{X}, M_{\mu+1} \subseteq\left\langle Y^{\prime \prime}\right\rangle+M_{\mu} \subseteq\left\langle Y^{\prime}\right\rangle+M_{\mu}$. Finally, if $\mu<\delta, y+g \in M_{\delta}$, so $y \in M_{\delta+1}$. But then $y \in M_{\delta}$ and hence $g \in\langle G\rangle \cap M_{\delta}=\left\langle G \cap M_{\delta}\right\rangle \subseteq\left\langle Y^{\prime \prime}\right\rangle$. Therefore $y+g \in\left\langle Y^{\prime \prime}\right\rangle$, and we are done since $Y^{\prime \prime}$ belongs to $\mathscr{X}$.

(e) First of all, we claim that if $\mathscr{X}^{\prime}=\mathscr{X} \mid A$ and if we are given a chain (7) up to $A$ which determines $\mathscr{X}^{\prime}$ as in (8), then (7) can be extended to a chain up to $M$ which determines $\mathscr{X}$. Indeed, if $(5)$ is the chain which demonstrates that $\mathscr{X}^{\prime}=\mathscr{X} \mid A$ (so that $A=M_{\mu}$ and $\left(5^{\prime}\right)$ determines $\mathscr{X}^{\prime}$ ), then one can easily check that the chain

$$
B_{0}<\cdots<B_{\rho}=A<M_{\mu+1}<\cdots<M_{\tau}=M
$$

determines $\mathscr{X}$. Now, using this claim, one can inductively define a continuous well-ordered chain $B_{0}<\cdots<B_{r}<\cdots$ so that for all $\nu<\tau, M_{\nu}=B_{r(\nu)}$ for 
some $\alpha(\nu)$ and $B_{0}<\cdots<B_{\alpha(\nu)}=M_{\nu}$ determines $\mathscr{X}_{\nu}$. This chain then shows that $\bigcup_{\nu<\tau} M_{\tau}$ belongs to $\mathscr{F}$, and determines $\mathscr{X}$, as described in (e).

Notice that Theorem 10 holds if in condition $(\mathrm{d}), \aleph_{0}$ is replaced by an infinite cardinal number $\mu$. If $\mathcal{N}$ is a set of $\mu$-presented modules and $\mathscr{F}_{\mathcal{N}}$ is defined as above for this $\mathscr{N}$, then Theorem 11 remains valid.

\section{Proof of Theorem A}

We are now ready to prove Theorem A stated in the Introduction.

First, the sufficiency of the condition is clear in view of [E1, p. 27].

To verify necessity, we apply induction on the cardinality $\kappa$ of a set of generators of the Baer module $B$. In case $\kappa \leq \aleph_{0}$, there is nothing to prove. Thus let $\kappa \geq \aleph_{1}$, and assume the claim is true for Baer modules with fewer than $\kappa$ generators.

Case 1. $\kappa$ is a regular cardinal. By Lemma 2 , we have p.d. $B \leq 1$, so $B$ has a tight system [FS, p. 84]. This is a collection $\mathscr{T}$ of submodules of $B$ such that (a) $0, B \in \mathscr{T}$; (b) $\mathscr{T}$ is closed under unions of chains; (c) if $B_{i}<B_{j}$ in $\mathscr{T}$, then p.d. $B_{j} / B_{i} \leq 1$; (d) if $B_{i} \in \mathscr{T}$ and $\Delta$ is a countable subset of $B$, then there is a $B_{j} \in \mathscr{T}$ such that $\left\langle B_{i}, \Delta\right\rangle \leq B_{j}$ and $B_{j} / B_{i}$ is countably generated. Hence we derive at once that for $B=M$ there is a sequence (1) satisfying conditions (i)-(iii) in Lemma 9. For the application of Lemma 9, choose $G_{\alpha}$ to be the direct sum of as many copies of $\bigoplus_{0 \neq r \in R} R / R r$ as the minimal cardinality of generating systems of $M_{\alpha+1} / M_{\alpha}$. If $B$ is a Baer module, then the set $E$ defined in Lemma 9 is not stationary in $\kappa$; thus there is a club $C$ in $\kappa$ which fails to intersect $E$. Keeping only the $M_{\alpha}$ 's with $\alpha \in C$ and renaming the elements of $C$ by the ordinals $<\kappa$, we obtain a chain (1) such that

$$
\operatorname{Ext}_{R}^{1}\left(M_{\alpha+1} / M_{\alpha}, H_{\alpha+1} / H_{\alpha}\right)=0
$$

for all $\alpha<\kappa$. Because of the choice of $G_{\alpha}$, Lemmas 4 and 5 guarantee that $M_{\alpha+1} / M_{\alpha}$ is a Baer module for each $\alpha<\kappa$. But all these $M_{\alpha+1} / M_{\alpha}$ are less than $\kappa$-generated; so the induction hypothesis applies, and we can insert between $M_{\alpha}$ and $M_{\alpha+1}$ a continuous well-ordered ascending chain of submodules with countably generated factors which are all Baer modules. If this is done for each $\alpha<\kappa$, we finally get a chain as stated in the theorem.

Case 2. $\kappa$ is a singular cardinal. Let $\mathcal{N}$ be the set of all countably generated Baer $R$-modules and let $\mathscr{F}=\mathscr{F}_{1}$ as defined before Theorem 11. Since every countably generated Baer module is countably presented by Corollary 7 , Theorem 11 applies and the prerequisites for Theorem 10 are satisfied. Now, let $\mathscr{T}$ be a tight system for $B$. For any $\kappa<\lambda$, let $\mathscr{C}_{\kappa}$ consist of all members of $\mathscr{T}$ which are $\kappa$-generated. Then by inductive hypothesis, $\mathscr{C}_{\kappa} \subseteq \mathscr{F}$, and by definition of a tight system, conditions $\left(2^{\prime}\right)$ and $\left(3^{\prime}\right)$ of Theorem 10 are satisfied. Hence, by Theorem $10 B \in \mathscr{F}$, which is the desired conclusion. 


\section{Proof of Theorem B}

Recall that a domain $R$ is called $h$-local $[\mathrm{M}]$ if (1) each nonzero prime ideal of $R$ is contained in only one maximal ideal of $R$, and (2) every nonzero element of $R$ is contained in only a finite number of maximal ideals of $R$. As is shown by Matlis [M, p. 47], $R \quad h$-local implies that every torsion $R$ module $T$ has a direct decomposition $T=\bigoplus_{P} T_{P}$ where $T_{P}=R_{P} \otimes T$ is the localization of $T$ at the maximal ideal $P$ of $R\left(T_{P}\right.$ may be called the $P$ component of $T$ ). Let $C(P)$ denote the complement $R \backslash P$; this is a semigroup under multiplication. Notice that $T_{P}$ is both $C(P)$-torsion-free and $C(P)$ divisible, while it is $C\left(P_{1}\right)$-torsion for every maximal ideal $P_{1} \neq P$. It is easily seen that the annihilator of each element in $T_{P}$ is contained in $P$. It follows at once from (2) that if $P_{n}(n=1,2, \ldots)$ are different maximal ideals of $R$ and $T_{P_{n}} \neq 0$, then the direct product $\prod T_{P_{n}}$ is not a torsion $R$-module.

Proof. The proof of Theorem B start with the finite rank case. So assume that $R$ is an $h$-local Prüfer domain and $B$ is a finite rank Baer module over $R$. Let $F$ be a free submodule of $B$ such that $B / F$ is torsion. Evidently, for any maximal ideal $P$ of $R$, the localization $B_{P}=R_{P} \otimes B$ has to be a Baer module over the valuation domain $R_{P}$. By [EF], $B_{P}$ is a free $R_{P}$ module. The exact sequence $0 \rightarrow F \rightarrow B \rightarrow B / F \rightarrow 0$ yields the exact sequence $0 \rightarrow F_{P} \rightarrow B_{P} \rightarrow(B / F)_{P} \rightarrow 0$ (recall that $R_{P}$ is a flat $R$-module); thus $(B / F)_{P}$ is a finitely presented torsion $R_{P}$-module.

If $(B / F)_{P} \neq 0$, then there exists a torsion $R_{P}$-module $T_{P}$ such that we have $\operatorname{Ext}_{R_{P}}^{1}\left((B / F)_{P}, T_{P}\right) \neq 0$. Hence $\operatorname{Ext}_{R}^{1}\left((B / F)_{P}, T_{P}\right) \neq 0$ as well. If $P_{1} \neq P_{2}$, then $\operatorname{Ext}_{R}\left((B / F)_{P_{1}}, T_{P_{2}}\right)=0$ since the first module is bounded $C\left(P_{2}\right)$-torsion, while the second is $C\left(P_{2}\right)$-torsion-free and $C\left(P_{2}\right)$-divisible. Putting $T=\bigoplus T_{P}$, we have

$$
\operatorname{Ext}_{R}^{1}(B / F, T)=\prod_{P} \operatorname{Ext}_{R}^{1}\left((B / F)_{P}, T_{P}\right),
$$

where the $P$ th component is a torsion $R_{P}$-module. By the remark in the last but one paragraph, the last product is not a torsion $R$-module unless almost all components vanish.

From the exact sequence $0 \rightarrow F \rightarrow B \rightarrow B / F \rightarrow 0$ we obtain the exact sequence

$$
\operatorname{Hom}_{R}(F, T) \rightarrow \operatorname{Ext}_{R}^{1}(B / F, T) \rightarrow \operatorname{Ext}_{R}^{1}(B, T)=0 .
$$

Here the first module is torsion, so the second is likewise torsion. But as we saw, this can happen only if $B / F$ has but finitely many nonzero $P$-components. Each $P$-component being finitely generated over $R$ as well, we argue that $B$ is finitely generated. Consequently, $B$-as a finitely generated torsion-free $R$ module-is projective.

In view of Theorem $\mathrm{A}$, in order to complete the proof of Theorem B, it clearly suffices to prove that over an $h$-local Prüfer domain $R$, a Baer module 
$B$ of countably infinite rank is projective. Evidently, such a $B$ is the union of a countable ascending chain $0=B_{0}<B_{1}<\cdots<B_{n}<\cdots$ of submodules where, for each $n, B_{n}$ has rank $n$ and $B / B_{n}$ is torsion-free. $R$ being a Prüfer domain, the last property implies $B_{n}$ pure in $B$. By Corollary 8, each $B_{n}$ is a Baer module and thus finitely generated projective, as shown above. $B_{n+1} / B_{n}$ as a finitely generated torsion-free module is projective. This means that $B_{n}$ is a summand of $B_{n+1}$, i.e., $B_{n+1}=B_{n} \oplus C_{n}$ for some necessarily projective submodule $C_{n}$ of $B_{n+1}$. Therefore $B$ is the direct sum of the $C_{n}$ 's, so projective, completing the proof of Theorem $B$.

\section{INDEPENDENCE RESULTS}

We want to consider whether Lemma 5 can be improved. Specifically, letting

$$
T_{0}=\bigoplus_{\omega} \bigoplus_{0 \neq r \in R} R / R r
$$

is it the case that $B$ is a Baer module if $\operatorname{Ext}_{R}^{1}\left(B, T_{0}\right)=0$ ? We know, by Lemma 5 , that the answer is "yes" if $B$ is countably generated. It turns out that in general, the answer to this question is independent of ZFC; this is a consequence of Theorems $C$ and 14. (For $R=\mathbb{Z}$, these are proved in [E2].)

Proof of Theorem C. This is by induction on the minimal cardinality $\kappa$ of a set of generators for $M$. If $\kappa=\aleph_{0}$, this is Lemma 5. So suppose $\kappa$ is uncountable, and the theorem has been proved for modules which can be generated by fewer than $\kappa$ generators.

Case 1. $\kappa$ is regular. As in the proof of Theorem A, the existence of a tight system for $M$ implies that there is a sequence (1) satisfying conditions (i)-(iii) of Lemma 9. Then by the proof of Theorem 1.5 of [E2], there is a club $C$ in $\kappa$ so that for $\nu \in C, \operatorname{Ext}_{R}^{1}\left(M_{\mu} / M_{\nu}, T_{0}\right)=0$ for all $\mu>\nu$. But then, by induction, for every torsion module $T$ and all $\mu>\nu \in C, \operatorname{Ext}_{R}^{1}\left(M_{\mu} / M_{\nu}, T\right)=$ 0 . Hence, by [E1, p. 27], $\operatorname{Ext}_{R}^{1}(M, T)=0$.

Case 2. $\kappa$ is singular. Since p.d. $M \leq 1, M$ has a tight system $\mathscr{T}$. For every $N \in \mathscr{T}, \operatorname{Ext}_{R}^{1}\left(N, T_{0}\right)=0$ (cf. the proof of Lemma 3 in [EF]). If $N \in \mathscr{T}$ is less than $\kappa$ generated, $N$ is a Baer module, by induction, and hence satisfies the conclusion of Theorem A. Then by the proof of the singular case of Theorem A, $M$ satisfies the conclusion of Theorem A, and is therefore a Baer module.

Now we turn to using the Proper Forcing Axiom to prove the opposite of what we proved in the last theorem.

Recall that a ring $R$ is called left perfect if every flat left $R$-module is projective. If $R$ is not left perfect, than there is an infinite descending sequence of principal right ideals

$$
r_{0} R>r_{0} r_{1} R>\cdots>r_{0} r_{1} \cdots r_{n} R>\cdots .
$$


Let $F$ be the free module on a basis $\left\{g_{n} \mid n \in \omega\right\}$ and $K$ the free submodule with basis $\left\{g_{n}-r_{n} g_{n+1} \mid n \in \omega\right\}$. Set $J=F / K$. The following is proved in [EM2, Theorem VII.1.3]; see also [EM1, p. 97].

Proposition 12. If $R$ is a ring which is not left perfect, and if $E$ is a stationary subset of $\omega_{1}$ consisting of limit ordinals, then there is a module $M$ which is not projective and which is the union of a continuous well-ordered ascending chain

$$
M_{0}<M_{1}<\cdots<M_{\nu}<\cdots \quad\left(\nu<\omega_{1}\right)
$$

where each $M_{\nu}$ is free of countable rank and for all $\nu: \mu, M_{\mu} / M_{\nu}$ is free if $\nu \notin E$, and $M_{\mu} / M_{\nu}$ is isomorphic to $J \oplus R^{(\omega)}$ if $\nu \in E$.

If $R$ is a domain which is not a field, then $R$ is not perfect, since the quotient field $Q$ of $R$ is flat, but not projective. If $J$ is as defined above, then $J$ is a countably generated torsion-free $R$-module of rank 1 . Noticing that it has a set of generators $\left\{g_{n} \mid n \in \omega\right\}$ such that $r_{n} g_{n+1}=g_{n}$ where $r_{n} \neq 0$ are nonunits in $R$, the proof of the next lemma is the same as the proof of Lemma 6 in [EF].

Lemma 13. If $R$ is a domain, then $J$ has projective dimension 1 and is not a Baer module.

For the Proper Forcing Axiom (PFA), see [Me2].

Theorem 14. Assume PFA. Let $R$ be a countable domain which is not a field. Then there is an $\aleph_{1}$-generated module $M$ of $p . d . \leq 1$, such that $M$ is not a Baer module, but $\operatorname{Ext}_{R}^{1}\left(M, T_{0}\right)=0$.

Proof. Choose $E \subset \omega_{1}$ which is stationary and costationary, and let $M$ be as in Proposition 12. Then $M$ has projective dimension $\leq 1$ by Auslander's Lemma [FS, p. 73], since each quotient $M_{\nu+1} / M_{\nu}$ has p.d. $\leq 1$. But $M$ is not Baer by Lemma 9 (or Theorem A), since for $\nu \in E$ and $\mu>\nu, M_{\mu} / M_{\nu}$ is not Baer, by Lemma 13. So it remains to prove that $\operatorname{Ext}_{R}^{1}\left(M, T_{0}\right)=0$. This proof is essentially the same as the one in [Me1, pp. 274-276]. Given a short exact sequence

$$
0 \rightarrow T_{0} \rightarrow H \stackrel{\pi}{\rightarrow} M \rightarrow 0
$$

let $\mathbf{P}=\left\{\theta \mid \theta: M_{\nu+1} \rightarrow H\right.$ is a splitting of $\left(\pi \mid \pi^{-1}\left[M_{\nu+1}\right]\right)$ for some $\left.\nu<\omega_{1}\right\}$. Partially order $\mathbf{P}$ by $\theta_{1} \leq \theta_{2}$ if and only if $\theta_{2} \subseteq \theta_{1}$. For $\tau \in \omega_{1}$, let

$$
D_{\tau}=\left\{\theta \in \mathbf{P} \mid M_{\tau+1} \subseteq \operatorname{dom} \theta\right\} .
$$

It is easy to see that $D_{\tau}$ is dense in $\mathbf{P}$, i.e., for every $\psi \in \mathbf{P}$, there exists $\theta \in D_{\tau}$ such that $\theta \leq \psi$. If $\mathbf{P}$ is proper, then PFA says that there is a directed set $G \subseteq \mathbf{P}$ (i.e., if $\psi_{1}, \psi_{2} \in G$, there is $\theta \in G$ so that $\theta \leq \psi_{i}$ ) such that for all $\tau, D_{\tau} \cap G \neq \varnothing$. Then $\cup G$ is the desired splitting: $M \rightarrow B$.

So it remains to prove that $\mathbf{P}$ is proper. Choose $\kappa$ so that $\mathbf{P}$ and $\mathscr{P}(\mathbf{P}) \in$ $H(\kappa)$ (the set of sets of hereditary size $<\kappa$ ). We need to prove that there is a club $\mathscr{C}$ of countable elementary substructures $\mathscr{N}$ of $H(\kappa)$ such that for all 
$\mathscr{N} \in \mathscr{C}$ and $\theta \in \mathbf{P} \cap \mathcal{N}$, there is $q \leq \theta$ such that $q$ is $\mathscr{N}$-generic, i.e., for every $D \in \mathscr{N}$ which is a dense subset of $\mathbf{P}$ and every $r \leq q$ there are $r^{\prime} \in D \cap \mathcal{N}$ and $p \in \mathbf{P}$ such that $p \leq r$ and $p \leq r^{\prime}$.

Let $\mathscr{C}$ consist of all countable elementary substructures $\mathscr{N}$ of $H(\kappa)$ such that $\mathscr{N}=\bigcup_{i \in \omega} \mathscr{N}_{i}$ where $\mathscr{N}_{i}$ is an elementary substructure of $\mathscr{N}_{i+1}$ and of $\mathscr{N}$, $\omega_{1} \cap \mathscr{N}_{i}$ is an ordinal $<\omega_{1} \cap \mathscr{N}_{i+1}$ and $\omega_{1} \cap \mathscr{N}_{i} \notin E$. Moreover we require that $\mathbf{P}, M$, and $\left\{M_{\nu} \mid \nu \in \omega_{1}\right\}$ belong to $\mathscr{N}_{i}$. Given $\mathscr{N} \in \mathscr{C}$ and $\theta \in \mathbf{P} \cap \mathcal{N}$, let $\mathscr{N}_{i}(i \in \omega)$ be as above, and define the ordinals $\beta_{i}=\omega_{1} \cap \mathcal{N}_{i}, \beta=\omega_{1} \cap \mathscr{N}$. We shall consider the case when $\beta \in E$, for it is the harder case. Let $\left\{D_{n} \mid n \in \omega\right\}$ be an enumeration of the dense subsets of $\mathbf{P}$ which belong to $\mathscr{N}$. Without loss of generality we can assume $D_{n} \in \mathscr{N}_{n}$ and $\theta \in \mathscr{N}_{0}$. Let $G_{n}=M_{\beta_{n}}$. We can inductively define $A_{n}, B_{n}$ so that

$$
G_{n} \oplus A_{n}=M_{\beta+1} ; \quad G_{n} \oplus B_{n}=G_{n+1} ; \quad A_{n+1} \oplus B_{n}=A_{n} .
$$

In fact, if $A_{n}, B_{n-1}$ have been chosen, let $B_{n}=A_{n} \cap G_{n+1}$; since $\beta_{n} \notin$ $E, G_{n+1}$ is a summand of $M_{\beta+1}$, thus $B_{n}$ is a summand of $M_{\beta+1}$ and hence of $A_{n}$. Consequently, $A_{n}=A_{n+1} \oplus B_{n}$ for some $A_{n+1}$. Let $\left\{g_{i} \mid i \in \omega\right\}$ be such that $M_{\beta+1}=\left\langle M_{\beta} \cup\left\{g_{i} \mid i \in \omega\right\}\right\rangle$, where without loss of generality, $g_{n} \in A_{n}$. Let $\pi_{0}$ be the projection of $M_{\beta+1}$ on $B_{n}$ relative to $G_{n} \oplus A_{n+1}$. Now, exactly as on p. 276 of [Me1], we can define $q=\rho^{\prime}: M_{\beta+1} \rightarrow H$, which is an $\mathscr{N}$-generic element of $\mathbf{P}$ extending $\theta$.

Added in proof. It is proved in On Whitehead modules by P. Eklof and S. Shelah (to appear in J. Algebra) that it is independent of $\mathrm{ZFC}+\mathrm{GCH}$ whether there is a single test module for being Baer of any cardinality.

\section{REFERENCES}

[B] R. Baer, The subgroup of the elements of finite order of an abelian group, Ann. of Math. (2) 37 (1936), 766-781.

[E1] P. C. Eklof, Set theoretic methods in homological algebra and abelian groups, Presses de l'Univ. de Montréal, Montreal, 1980.

[E2] _-, Homological algebra and set theory, Trans. Amer. Math. Soc. 227 (1977), 207-225.

[EF] P. C. Eklof and L. Fuchs, Baer modules over valuation domains, Ann. Mat. Pura Appl. 150 (1988), 363-373.

[EM1] P. Eklof and A. Mekler, Categoricity results for $L_{\infty \kappa^{-}}$-free algebras, Ann. Pure Appl. Logic 37 (1988), 81-99.

[EM2] _ Almost free modules: set-theoretic methods, North-Holland.

[FS] L. Fuchs and L. Salce, Modules over valuation domains, Lecture Notes in Pure and Appl. Math., Vol. 97, Marcel Dekker, 1985.

[Gö] R. Göbel, New aspects for two classical theorems on torsion splitting, Comm. Algebra 15 (1987), 2473-2495.

[Gf] P. Griffith, A solution to the splitting mixed problem of Baer, Trans. Amer. Math. Soc. 139 (1969), 261-269.

[Gm] R. Grimaldi, Baer and LT-modules over domains, Ph.D. Thesis, New Mexico State Univ., 1972. 
[H] W. Hodges, In singular cardinality, locally free implies free, Algebra Universalis 12 (1981), 205-220.

[K] I. Kaplansky, The splitting of modules over integral domains, Arch. Math. 13 (1962), 341343.

[M] E. Matlis, Cotorsion modules, Mem. Amer. Math. Soc. No. 49 (1964).

[Me1] A. Mekler, Proper forcing and abelian groups, Abelian Group Theory, Lecture Notes in Math., Vol. 1006, Springer-Verlag, 1983, pp. 285-303.

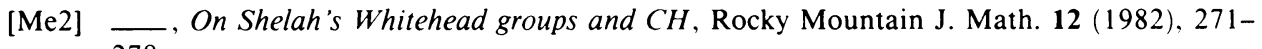
278.

[N] R. J. Nunke, Modules of extensions over Dedekind rings, Illinois J. Math. 3 (1959), 222-241.

[O] B. Osofsky, Upper bounds on homological dimensions, Nagoya Math. J. 32 (1968), 315-322.

[S] S. Shelah, Incompactness in regular cardinals, Notre Dame J. Formal Logic 26 (1985), 195-228.

Department of Mathematics, University of California, Irvine, California 92717

Department of Mathematics, Tulane University, New Orleans, Louisiana 70118

Department of Mathematics, The Hebrew University, Jerusalem, IsRael 